\title{
Nonseparability of Shared Intentionality
}

\author{
Christian Flender, Kirsty Kitto and Peter Bruza \\ Faculty of Science and Technology, \\ Queensland University of Technology, \\ Brisbane, Australia \\ \{c.flender, kirsty.kitto, p.bruza\}@qut.edu.au
}

\begin{abstract}
According to recent studies in developmental psychology and neuroscience, symbolic language is essentially intersubjective. Empathetically relating to others renders possible the acquisition of linguistic constructs. Intersubjectivity develops in early ontogenetic life when interactions between mother and infant mutually shape their relatedness. Empirical findings suggest that the shared attention and intention involved in those interactions is sustained as it becomes internalized and embodied. Symbolic language is derivative and emerges from shared intentionality. In this paper, we present a formalization of shared intentionality based upon a quantum approach. From a phenomenological viewpoint, we investigate the nonseparable, dynamic and sustainable nature of social cognition and evaluate the appropriateness of quantum interaction for modelling intersubjectivity.
\end{abstract}

\section{Introduction}

How do we relate to others and how do social interactions shape our worldview? What are the processes underlying everyday social encounters and how do these processes contribute to enculturation and our use of symbolic language? Questions of this kind have been of considerable interest ever since the social world became the object of investigation. More recently, studies in developmental psychology and cognitive neuroscience have shed new light on how we interact with each other and the world we inhabit. In conjunction with phenomenological descriptions, potential scientific explanations of intersubjectivity emphasize the role of social perception and the importance of sustained interactions between social beings. From the earliest age, human infants relate to their mothers in an embodied and imitative way which lays the foundation for them to grow into social and cultural reality. The distinction of self and other as well as linguistic abilities are claimed to emerge from an ongoing interaction with other humans 112341516 .

In adulthood, developmental perspectives also provide evidence of how we are able to adopt a detached or third person view of the world. Experiencing concrete categories like book or abstract concepts like democracy as distinct or independent from us as observers, is like these objects being there for everyone 7889. If we perceive others, for instance in a conversation, we do this by presupposing our partners to be subjects like us, but in a way that transcends our 
subjectivity due to the other being there for, or accessible to, everyone else. It is this presupposed intersubjectivity that facilitates a shared world of objects as the foundation for enculturation including the development of symbolic language.

Language and the sense of self and other develop in early ontogenetic life through an ongoing interaction between mother and infant. Even before the infant develops a deliberate sense of self, there is a bodily pairing between mother and infant that is characterized by an intermodal link between action, in particular motor behaviour, and perception of the mother 1011. It is this perceptual, practical and self-enganging sense of other agents which is essentially not separable into ego and alter ego (self and other) and maintained throughout life. There is always some degree of undifferentiated identification; self and other are never fully distinguished [12].

In this paper, we want to make explicit this presuppositional sense of the other by formalizing shared intentionality using notions borrowed from quantum mechanics. Quantum formalisms lend themselves to model interactive, contextdependent and emerging phenomena [13/1415]16] and could provide considerable help in developing a more precise understanding of intersubjectvity.

We proceed as follows. In the next section, we introduce intersubjectivity by means of two important concepts. Firstly, social perception emphasizes the direct or non-inferential character of social interaction. Secondly, the discovery of mirror neurons in cognitive neuroscience supports social perception. Mirror neurons provide an intermodal bridge between action and perception. Acting and perceiving someone else performing are two nonseparable and intentional concepts which we will formally introduce in Section 3. We will show that states involved in mother-infant interactions are essentially nonseparable and that this can be represented as a an entangled quantum state. The mutual anticipation of the other's reaction involves nonseparable states of an emerging interaction process. In Section 4, we conclude that entangled states must derive from the nonseparable time evolutions that govern the dynamic co-emergence [17] of shared intentional states and intentional states of mother and infant. Departing from our developed notion of shared intentionality, in Section 5, we disuss intersubjectivity from the perspective of phenomenology. We contrast intersubjectivity as empathy with intersubjectivity as co-subjectivity and integrate both dimensions under the umbrella of shared intentionality. Lastly, we give a conclusion and outlook toward future work.

\section{Intersubjectivity}

In this section, we introduce intersubjectivity or shared intentionality according to recent discussions in developmental psychology and neuroscience. In sharing intentions, agents perceive each other based upon a multiplicity of states such as emotions or somatic sensations [18. Mirror neurons support the nonseparability of such states as they link one's own actions with perception of someone else's movements and actions. 


\subsection{Social Perception}

Classical theories of social cognition have been critizised for overintellectualizing social cognition by underestimating perception [10 19/20. The two dominant theories, Theory theory (TT) and simulation theory (ST), require subjects to add extra inferential mechanisms in order to understand the other. However, experiments reveal infants reacting with facial expressions or patterns of vocalization and gestures to affordances such as movement or sounds without a need for theory or models $[19$.

Once the child develops and acquires concepts, the initial smartness of early infant perception (that is perception as inherently active, direct and non-inferential) is maintained [10/1|21|22]. I still recognize my friend as being my friend without necessarily attributing him with a certain attitude towards me. Hence, neither the smartness nor the directness of perception is necessarily dependent on perceiving things under concepts or judgement [10. Put another way, perception itself is conceptual but not in the sense of explicit deliberate judgement [23]. Perception essentially gets shaped or informed by the ongoing interaction between intentionalities 20]. Of course, the role of perception does not rule out deliberation affecting perception, however, in many social encounters the smartness of direct perception mirrors our ability to skilfully interact with others.

Later in ontogenetic life, perception is also informed by the language and concepts we learn. Empirical studies show that children acquire linguistic abilities through language use 3121] and thus through social interaction. Obviously, this presupposes the social community the child is part of. Moreover, the community itself embodies conventionalized conceptual structure derived from language use. Learning words intersubjectively in early ontogenetic life is essentially a process of extracting elements from the larger linguistic construction of adults. After months of gestural and vocal interaction, most Western middle-class children begin producing linguistic utterances in the months following the first birthday [1]. Such first expressions (holophrases), which are mainly declarative statements, imperative requests and interrogative questions, are learned and used in the same intentional context as for the perceptual or non-linguistic intentionalities during the first year of their life. From there, more complex constructions develop. For instance, conceptual integration, the formation of abstract concepts accross episodes or the reflexive adoption of the perspective of the listener. It is the latter which has only recently been taken seriously into account.

\subsection{Mirror Neurons}

A phenomenology of intersubjectivity reveals that social interaction is often not inferential. Others have argued against explicit deliberate judgement or deductive reasoning as the only form of sense-making 2425]26. When we are absorbed in complementary communicative gestures, dance-like behaviours or language games (in a Wittgensteinian sense), there is no need for detached and deliberate reasoning. However, this does not deny complex processes on a subpersonal or neural level. On a sub-personal or unconsciouss level, mirror neurons 
are activated both when an agent acts and when this agent perceives someone else acting [27. Mirror neurons integrate both motor and perceptual properties, i.e. they tightly couple observed behaviour with one's own performance. For instance, in monkeys cells discharge when an experimenter places food in front of the monkey and when the monkey reaches for it 28. More recently, mirror neurons have received much scientific interest since they do not just mirror observed physical behaviour, but purposeful action. Hence, they support understanding others to be like oneself. Furthermore, it was shown that mirror neurons are not just receptive to the teleological structure of an action, but also to the style or manner of the action in which the goal is achieved [29]. Although mirror neurons were first discovered in the brain area F5 of the ventral premotor cortex of monkeys, there is strong evidence for their existence in humans [28|29|30. Even more striking is that area F5 in monkeys is homologous to Broca's area in humans, a part of the brain associated with linguistic ability and verbal communication 29131.

In mother-infant interactions, it has been argued that the mirror system is a necessary though not sufficient condition for imitation [30. From an early age on children perceive intentions of their mother. In their ability to imitate behaviour, an ability in which humans differ from monkeys and other primates [19, children directly perceive the other without consciously inferring what the mother's goals are. Direct perception in social interaction is facilitated through an intermodal bridge between observing the other and enacting what the other does. Perception of others and motor action are nonseparably mapped through the underlying mirror system [3010].

\section{Sharing Intentions}

Infants responsiveness to facial expressions, gestures or sounds and their ability to imitate is not based upon inference, analogy or simulation. It is the direct perception of others and the intentional potentialities of their own body that facilitates understanding others as animate beings like themselves [11]. Mirror neurons are supportive of this view since they provide an intermodal and nonseparable link between action and perception. In the following, we develop a formal model of the concepts introduced in the previous section. We start with the notion of intentionality following the phenomenological tradition of Edmund Husserl [32 9]33.

\subsection{Intentionality}

According to Husserl, human experience is intentional, i.e. it aims toward something beyond itself. This means that every experienced phenomenon is about, or of something, i.e. it is directed. For instance, the infant's perception of its mother or the mother's imagination of her child's well being are intentionally directed experiences. Moreover, intending something is always accompanied by certain flavours such as bodily sensations or moods but also communal norms, 
conventions and historical traditions. For instance, the infant might be hungry while perceiving its mother or the mother might be depressed while imagining her child being sick. Those constituents of experience are not directed and thus open. According to Husserl, many of such presupposed meanings are tacitly taken over from our culture and belong to the so-called lifeworld (Lebenswelt).

Directedness. Intentional experiences are directed though not necessarily toward an object that is explicitely distinguished. For instance, the mother can directly perceive her infant as being her child or she can deliberately reason about the amount of time the child kept sleeping last night. No matter what degree of awareness, directed experience is inherently temporal and has a correlational structure. This structure inseparably connects intentional act (noesis) and sense or appearance of an object (noema). Noemata correspond to all anticipations we have about, or of, an object. For instance, in perceiving her infant the mother presupposes it to be her child. She does not explicitely judge but rather implicitly anticipates the senses which constitute the relationship to her child. Noesis is the way or mode in which this anticipation takes place or unfolds. It discloses the noemata of an object in time and so gives meaning to it. For instance, if the mother sees her child crying, the mode, or way she anticipates her child changes toward a stronger bodily tension [4].

Let $B=\{|0\rangle,|1\rangle\}$ be the orthonormal basis of an intentional object. The orthonormal basis generates a vector space. Each vector represents a possible noema, sense or meaning in that space and can be written as a linear combination of orthogonal vectors. Let an intentional object be:

$$
|p\rangle=a_{1}|0\rangle+a_{2}|1\rangle
$$

where $\left|a_{1}\right|^{2}+\left|a_{2}\right|^{2}=1$. Noemata correspond to all anticipated senses of an intentional object while being inseparably connected to the way an object is disclosed. For instance, consider an infant's awareness of its mother as a superposition or anticipation of senses. The basis vector $|0\rangle$ stands for the primordial sense 'do not expect reaction', whereas $|1\rangle$ represents 'expect reaction'. As discussed in the previous section, perceiving others is not believing, thinking or reasoning. It is more to be drawn to do something, a tension that the body aims to reduce and where we do not pay attention to the anticipation itself [1121]. Let the probability of noesis to disclose noemata be defined as:

$$
P(|0\rangle)=\left|a_{1}\right|^{2} \text { and } P(|1\rangle)=\left|a_{2}\right|^{2}
$$

Noeses represent the degree of bodily tension or anticipation. For instance, the infant can have a strong or weak bodily tension towards its mother. Acts exhibit a certain probability $P$ and unavoidably disturb the intentional object leaving it in a state $|0\rangle$ or $|1\rangle$ determined by the outcome.

Openness. Open intentionality corresponds to Husserl's lifeworld (Lebenswelt) 9]. The lifeworld is always and already pregiven or presupposed and presents 
directed experience in a certain light. For instance, bodily sensations such as pain, moods such as happiness, or absorbed skillful activities such as driving and dancing are open, prepredicative and complement directed activities. They only take on an object-directed structure in moments of breakdowns, e.g. I attend to my hurting knee or the strange engine sound. Hence, open intentionality is subpersonal but can potentially be brought to awareness. It forms the horizon or ground of all our activities. Husserl considers intersubjectivity as crucial for the generation and transformation of this presupposed horizon [9]. In the next section we will formalize what happens if an infant's directedness towards its mother and vice versa becomes embodied or internalized and so a part of the lifeworld. Anticipations can become entangled and thus they can not be separated into directed intentionalities of either mother or infant.

\subsection{Shared Intentionality}

If mother and infant interact their intentionalities are directed towards each other. Vector $\left|p_{1}\right\rangle$ represents the mother's awareness of her infant. $\left|p_{2}\right\rangle$ represents the infant's awareness of its mother.

$$
\left|p_{1}\right\rangle=a_{1}|0\rangle+a_{2}|1\rangle \text { and }\left|p_{2}\right\rangle=b_{1}|0\rangle+b_{2}|1\rangle
$$

where $\left|a_{1}\right|^{2}+\left|a_{2}\right|^{2}=1$ and $\left|b_{1}\right|^{2}+\left|b_{2}\right|^{2}=1$. Vectors $\left|p_{1}\right\rangle$ and $\left|p_{2}\right\rangle$ have a tension towards each other. To make this clear, we define shared intentionality as a larger combined vector space. Here, $S=B_{M} \otimes B_{I}=\{|00\rangle,|01\rangle,|10\rangle,|11\rangle\}$ is the basis of mother and infant. In this combined space, shared senses $|\psi\rangle$ can be generated.

$$
\begin{aligned}
|\psi\rangle & =\left|p_{1}\right\rangle \otimes\left|p_{2}\right\rangle \\
& =\left(a_{1}|0\rangle+a_{2}|1\rangle\right) \otimes\left(b_{1}|0\rangle+b_{2}|1\rangle\right) \\
& =a_{1} b_{1}|00\rangle+a_{2} b_{1}|10\rangle+a_{1} b_{2}|01\rangle+a_{2} b_{2}|11\rangle
\end{aligned}
$$

where $\left|a_{1} b_{1}\right|^{2}+\left|a_{2} b_{1}\right|^{2}+\left|a_{1} b_{2}\right|^{2}+\left|a_{2} b_{2}\right|^{2}=1$. However, experiments have shown that an infant's anticipation of its mother is not the crucial point [5]. Infant and mother rely on each other to behave responsively in order to sustain their involvement in interaction 34/6. Two-month-old infants are able to sustain interaction with their mothers via a live double video link. However, when they are shown recordings of their mothers, they do not coordinate with the unresponsive recording but become distressed and removed. More important is the ongoing anticipation of the mother. The infant performs some actions and anticipates the mother's reactions reflecting the infant's actions and vice versa, i.e. the mother's anticipation of the infant's reactions. Sustained social interactions can only be established when these anticipations are mutual and dynamic [35. Therefore, intentionalities of mother and infant can not be separated and should be represented as an entangled or nonseparable state.

$$
|\psi\rangle=x|00\rangle+y|11\rangle
$$


If $|\psi\rangle$ is entangled, there is no $\left|p_{1}\right\rangle$ and $\left|p_{2}\right\rangle$ such that $|\psi\rangle=\left|p_{1}\right\rangle \otimes\left|p_{2}\right\rangle$. Since $|\psi\rangle$ is not separable into directed intentionalities of mother and infant, it requires both having access to it and thus $|\psi\rangle$ is open. Anticipations of mother and infant (4) evolve towards undirected intersubjectivity (5) which becomes part of the presupposed lifeworld of mother and infant. Crucial is the ongoing interaction that sustains mother's and infant's directedness toward each other. This process does not only emerge from the mutual anticipation of mother and infant, it also shapes the lifeworld of both as shared intentionality becomes internalized and embodied. Hence, individual intentionalities can not be defined independently of the emerging interaction process and the entangled social system can not be defined independently of the single agents. Shared intentional states (5) and directed intentional states of the individuals (1) co-emerge by co-enacting each other [20]22]. Therefore, senses or appearances of an agent, so far represented as state vectors, depend on shared intentional states as much as shared appearances rely on senses of the individual agents. To make this more precise, we need to have a look at shared intentionality evolving in time. Let the time evolution of mother's and infant's directed intentionality be defined as:

$$
U_{M}=e^{i A_{1} t} \text { and } U_{I}=e^{i A_{2} t}
$$

The time evolution consists of the exponentiation operation $e$, a self-adjoint operator $A$ and a time parameter $t$. Similar to intentional objects (4), time evolutions of two or more agents can be combined by using the tensor product. The only difference is that combined evolutions are products of tensor-product evolutions and not superpositions of tensor-product evolutions [17.

$$
\begin{aligned}
U_{M+I} & =e^{i A_{1+2} t} \\
& =e^{i\left(a_{1} b_{1} \rho_{1}+a_{2} b_{1} \rho_{2}+a_{1} b_{2} \rho_{3}+a_{2} b_{2} \rho_{4}\right) t} \\
& =e^{i a_{1} b_{1} \rho_{1} t} e^{i a_{2} b_{1} \rho_{2} t} e^{i a_{1} b_{2} \rho_{3} t} e^{i a_{2} b_{2} \rho_{4} t}
\end{aligned}
$$

Since our claim is that agents evolve from the background of an always and already pregiven intersubjective lifeworld, states or senses of intentional objects are now represented as density operators $\rho$; senses are always and already intersubjective and thus combine 1 . The density operators $\rho_{1-4}$ represent the basis vectors in $S$ and thus the state vectors or senses of $B_{M}$ and $B_{I}$. The evolution equation can be written as a product because the members of $S$, i.e. the basis states of mother and infant, are mutually commuting. From a mathematical point of view $U_{M+I}$ derives from the basis states in $S$. However, experiments reveal that mother-infant interactions are mutual and dynamic. Therefore, time evolutions $U_{M}$ and $U_{I}$ of the density operators representing mother and infant can not be characterized independently of $U_{M+I}$. For instance, consider the mother's

\footnotetext{
${ }^{1}$ In this way we can represent both experiences being intersubjectively open and thus nonseparable (e.g. perceiving someone as being there for, or accessible to, me and others) and intentional objects appearing as distinct and thus separable (e.g. perceiving someone as being transcendent and thus distinct from me and others) [ 8 .
} 
awareness of her infant while perceiving her child as a density operator ${ }^{2}$

$$
\begin{aligned}
\rho & =\operatorname{Tr}(\rho(t)) \\
& =a_{1}(t)|0\rangle\left\langle 0\left|+a_{2}(t)\right| 1\right\rangle\langle 1|
\end{aligned}
$$

where $a_{1}(t)=|\langle 0(t) 0 \mid \psi\rangle|^{2}$ and $a_{2}(t)=|\langle 1(t) 1 \mid \psi\rangle|^{2}$. If, from her first-person perspective, the mother does not expect her infant to react at time $t$, then $|0(t)\rangle=U_{M}(t)|0\rangle$ and if she expects a reaction, then $|1(t)\rangle=U_{M}(t)|1\rangle$. Although social perceptions are intersubjective and thus open, agents are not always involved in sustained face-to-face interactions. If the mother perceives her child sleeping, her directed experience does not rely on the infant reacting to her perceptions. In this case, $U_{M}$ and $U_{I}$ are separated and perceiving her child sleeping depends on the mother's private experience at each instant of time $\left(a_{1}(t)\right.$ and $\left.a_{2}(t)\right)$. Nevertheless, her experience is intersubjectively constituted and thus there is a suppositional reference to nonseparable states $|\psi\rangle$. If the unitary evolution of mother and infant is separable, $\psi$ is presupposed and not needed at each instant of time to describe their combined evolutions; there is no mutual face-to-face encounter. However, as experiments have shown, if motherinfant expectations are mutual and dynamic, $U_{M+I}(t)(7)$ is nonseparable, i.e. it can not be factorized into the tensor product $U_{M}(t) \otimes U_{I}(t)$. Consequently, nonseparable states dynamically emerge from mutual anticipations and can not be defined independently of mother and infant. Hence, expectation values of senses to be disclosed from the first-person perspective of mother and infant also rely on shared senses at each instant of time. Therefore, $a_{1}(t)=|\langle 00 \mid \psi(t)\rangle|^{2}$ and $a_{2}(t)=|\langle 11 \mid \psi(t)\rangle|^{2}$. Compared to shared intentional states (5), the ongoing interaction between mother and infant, i.e. the nonseparable time evolution, provides a higher degree of nonseparability since the nonseparable state $|\psi\rangle$ is needed at each instant of time to describe the evolution of mother's and infant's directed intentionality towards each other. Shared intentional states emerge from social interactions but also submerge or modulate individual agents. Subjectivities of both mother and infant move quite literally as a whole. In the remainder we will consider the possibility of this nonseparability of shared intentionality being not only relevant to face-to-face encounters but also to intentionality-tolifeworld relations in general.

\section{Discussion}

In this section we discuss shared intentionality from a phenomenological perspective. Intersubjectivity or shared intentionality is considered as a constitutive aspect of phenomenal experience. An agent's experience is analysed in terms of conditions of possibility for manifestation. In other words, we examine the role of intersubjectivity as a condition for animate beings and inanimate objects to become manifest in experience. We divide our discussion of intersubjectivity in

\footnotetext{
${ }^{2}$ Note that senses, now represented as density operators, derive from the dynamic co-emergence [17]. Senses are transcendental or inexhaustible $36[22$.
} 
two parts. Firstly, we look at face-to-face encounters like the mother-infant interaction presented in the previous section (empathy). Secondly, we examine the disclosure or manifestation of intentional objects precisely as being there for, or accessible, to others (co-subjectivity). We will argue that both dimensions of intersubjectivity can be understood under the umbrella of shared intentionality as developed in the previous section.

\subsection{Empathy}

One of the core problems of phenomenological intersubjectivity is the question of how we can have access to other minds [37. In particular, one of the most intriguing questions is the relation between empathy, the experience of otherness, and our existence in a common or shared world. We shall focus on empathy first. Empathy is a form of social perception enacted in face-to-face encounters and directed toward the experience of the other. Contrary to the argument from analogy $y^{3}$ empathy is inherently active, direct and non-inferential (cf. section 2). Therefore, perceiving others is not attributing internal mental states inferred from observed external behaviour rather behaviour as perceived is expressive. According to Scheler (1954), expressive behaviour is neither perceived as a mere body nor as a hidden psyche but as a unified whole [12. Furthermore, perceiving others as animate beings is different from perceiving physical objects. The other is given as bodily present or as a lived body (Leib) and not just as a transcendent object (Körper).

As defined in section 3, empathy is an intentional act that is directed towards the other's lived experiences. In this dynamic process, subjectivity of the other is disclosed from the second-person perspective. The second-person perspective is one's own open lived experience directed toward the open and directed lived experience of the other. Obviously, this nonseparability of first-person perspective and second-person view is reminiscent of the nonseparability of shared intentionality as introduced in the previous section. Crucially, empathy is not a multi-stage process where one observes mere external behaviour (behaviourism) and then adopts a theoretical stance to infer or compute the internal mental state of the other (cognitivism). As Heiddegger points out, grasping mental states of others is the exception rather than the rule. Under normal circumstances we understand each other well enough through our shared engagement in the common world [38. This preflecitve otherness (alterity) accompanying everyday social encounters is often called primary intersubjectivity 39].

Moreover, social interaction extends toward secondary intersubjectivity 40 or the ability to share attention and intention. Here, interacting partners do not only relate to each other but refer to objects and events around them. In such triadic situations, agents learn to understand other's intention by means of other's expressive and contextualized behaviour. For instance, gaze-monitoring indicates that agents seek to verify the attention of the other towards the same thing, e.g.

\footnotetext{
3 According to the argument from analogy, I infer by analogy that observed behaviour of foreign bodies is associated with experiences similar to those I have myself.
} 
a hammer lying on a table, as well as to validate whether their intention is understood and thus shared. Hence, intersubjectivity in social perceptions is not always exclusively directed towards others but often mediated through the pragmatic circumstances of our encounters. For instance, when perceiving a hammer and nails, I see those tools as affordances or possible uses which were essentially learned in an intersubjective and pragmatic context. Likewise, perceiving other agents is pragmatic and context-dependent. Here, affordances are possible intentions associated with the perception of the other embedded or situated in pragmatic contexual situations, e.g. I perceive my friend as someone who is an expert with tools like hammers and nails.

From a developmental perspective, Tomasello (1999) has proposed that we gradually develop our understanding of others starting from (1) animate beings (from birth onwards) over (2) intentional agents (9-12 months) to (3) mental agents (4-5 years) [2]. In the first stage, children solely empathize by perceiving expressive behaviour and so they can distinguish animate from inanimate beings. Approaching their first year of life, expressive behaviour is increasingly experienced as goal-directed and context-dependent. Phenomena such as gazefollowing, joint attention, shared engagement and imitative learning are indicators for children being able to see others as intentional agents. Apparently, these stages correspond to primary intersubjectivity and secondary intersubjectivity. The third stage essentially derives from social interactions in the previous stages. To understand others as mental agents requires to understand that others have beliefs and thoughts differing from one's own thoughts and beliefs. Children need to engage in discourses in which diverging perspectives emerge, e.g. disagreements, misunderstandings or requests for clarification. Importantly, understanding others as mental agents requires primary and secondary intersubjectivity upon which narrative competency and skilful practical reasoning develops [41]. However, narrative and practical reasoning skills do not involve reference to unobservable, abstract and general entities as postulated by some kind of theory of mind, e.g. TT. Rather such skills are grounded in observable events that take place in the world. The concrete and particular context is of primary importance for the determination of meaning [42].

In summary, children flourish starting with undifferentiated self-other relations or empathetic face-to-face encounters. From there, they engage in triadic communications laying the groundwork for experiencing others as situated and intentional beings. Eventually, children derive belief systems from language-use. Obviously, this presupposes the social community the child is a part of. Moreover, the community itself embodies conventionalized conceptual structure derived from language use. Therefore, even beyond our development in childhood, there is an always and already pregiven co-subjectivity, an anonynmous being-withothers, that is characteristic of intersubjectivity though different from empathy.

\subsection{Co-Subjectivity}

Empathy can be considered as a subtle form of intersubjectivity according to which an agent directly perceives expressive behaviour of other animate beings. 
However, the other's expressive behaviour is never isolated or separated. There is always and already a shared or common ground that influences how behaviour is perceived. Therefore, empathetically relating to others never requires a theoretical understanding, or model, of someone else's mind rather behaviour is expressive in the sense that a common lifeworld affords me to perceive certain shared aspects tuning the expressiveness of other's behaviour. As Husserl points out, I have been together with others for as long as I can remember, and my understanding and interpretation are, therefore, structured in accordance with intersubjectively handed-down forms of apperception 33. Obviously, drawing from communal norms, social conventions and historical traditions does not only structure how we empathize and perceive other social beings but shapes our intentional relation to the world, including inanimate things and abstract objects alike. For instance, when visually perceiving an object, e.g. a glass of red wine, the impoverished sense data on my retina does not cause me to internally reconstruct or represent the external object. The object is actively explored as a function of my body movement, a prereflective sensorimotor grasp, where qualities like shape and color of the glass are directly perceived rather than internally represented. We visually experience the world to be rich in detail not because we must represent all that detail inside our heads at any given moment, but because we have constant access to the presence and detail of the world, and we know how to make use of this access [23. In this way profiles hidden from view, e.g. the backside of the glass, are brought to awareness. Phenomenologically speaking, the glass is precisely given in experiences as being there for or accessible to others. As Zahavi (2006) puts it, subjects intentionally direct toward objects whose giveness in experience bears witness to their openness for other subjects [37. Husserl was quite clear about the intersubjective or co-subjective nature of experience. According to his analysis, everything objective that stands before me in experience and primarily in perception has an apperceptive horizon of possible experiences. Every appearance that I have is from the very beginning a part of an open endless, but not explicitely realized totality of possible appearances of the same, and the subjectivity belonging to this experiences is open intersubjectivity [9. Hence, appearances or senses are transcendental or inexhaustible (cf. equation 8). Consequently, even prior to empathetic interactions with other social beings, intersubjectivity is already present as co-subjectivity.

What is the relation between empathy and co-subjectivity? According to our model as developed in the previous section, emapthy and co-subjectivity are not separable. Empathy essentially refers to the co-directed intentionality that entangles with the pregiven co-subjectivity of the lifeworld. The most obvious form of empathetic relations is the sustained involvement in mutual anticipations. In our example, mother and infant empathize by continuously anticipating the other's directed awareness. The nonseparable time evolution that governs the coemergence of the interaction and modulation process literally moves as a whole when the mother's co-subjectivity (i.e. her intersubjectively handed-down forms of apperception) entangles with the subjectivity of the child. Presumably, shared intentionality is an important ontogenetic and phylogenetic process that shapes 
human consciousness. Neither empathy nor co-subjectivity derive from one another, rather empathy and co-subjectivity go hand-in-hand, they dynamically co-emerge in time.

\section{Conclusion and Future Work}

Intersubjectivity is a necessary condition for self and language to unfold. It develops in early ontogenetic life when interactions between mother and infant mutually shape their relatedness. We presented a model of shared intentionality that represents intersubjectivity as nonseparable or entangled states of an ongoing process of mutual anticipation. Entanglement is a phenomenon that only arises in quantum mechanics. Hence, a quantum model is necessary. It is the sustained involvement in interaction that leads to entangled and shared intentional states. These states are not separable and thus they are not reducible to directed intentionalities of either mother or infant. Moreover, it is the ongoing interaction between mother and infant that is neither reducible to subjectivity of mother nor to subjectivity of infant.

Generally, empathic skills facilitate the understanding of expressive behaviour in others. In addition, empathic skills lay the groundwork for sharing attention and intention and imitative learning, as well as linguistic abilities such as narrative and practical reasoning competencies. Once internalized and embodied, the shared intentional states involved in all of these social skills become essentially part of the lifeworld. The already and always pregiven lifeworld presupposes how objects including other subjects are disclosed in experience and in this way it presupposes noemata co-constituted by other subjects.

Despite this not being obvious from a naïve realist point of view, closer phenomenological examinations reveal experience including perception to be essentially intersubjective. From a first-person perspective, all observed phenomena, whether physical or not, are private. However, there are objects and events to which there is public access. These public phenomena are not transcendent in any subject-independent sense. They are objective precisely in the sense of open intersubjectivity as introduced in this paper. Essentially, there is no necessity for maintaining the dualism of subjectivity and objectivity since intersubjectivity or shared intentionality mediates the latter and includes the former 8 . This understanding of shared intentionality is further strengthened by recent findings in developmental psychology and neuroscience.

Departing from our proposed notion of shared intentionality and its nonseparable nature, future work will investigate how individual intentionalities are transformed and even generated in social interactions. Furthermore, one of the most interesting questions is how such an account deals with failures in everyday

social encounters. If breakdowns are mediated by the interaction process itself what are the consequences for understanding miscommunication?

Acknowledgements This project was supported in part by the Australian Research Council Discovery grant DP0773341. 


\section{References}

1. Tomasello, M.: Acquiring linguistic constructions. Handbook of Child Psychology 1 (2006) 1-48

2. Tomasello, M.: Constructing a Language: A Usage-Based Theory of Language Acquisition. Harvard University Press (2003)

3. Tomasello, M.: The Cultural Origins of Human Cognition. Harvard University Press (1999)

4. Gibbs, R.: Intention as emergent products of social interactions. In: Intentions and Intentionality: Foundations of Social Cognition. MIT Press (2001) 105-124

5. Murray, L., Trevarthen, C.: Emotional Regulation of Interactions between Twomonth-olds and their Mothers. In: Social Perception in Infants. Ablex: Norwood, NJ (1985) 177-197

6. Trevarthen, C.: The Self Born in Intersubjectivity: The Psychology of an Infant Communicating. In: The Perceived Self: Ecological and Interpersonal Sources of Self-knowledge. Cambridge University Press (1993) 121-173

7. Zahavi, D.: Beyond Empathy: Phenomenological Approaches to Intersubjectivity. Journal of Consciousness Studies 8 (2001) 151-168

8. Velmans, M.: Intersubjective Science. Journal of Consciousness Studies 6 (1999) 299-306

9. Husserl, E.: Husserliana 14: Zur Phänomenologie der Intersubjektivität. Texte aus dem Nachlass. Zweiter Teil: 1921-1928. Den Haag: Martinus Nijhoff (1973)

10. Gallagher, S.: Direct Perception in the Intersubjective Context. Consciousness and Cognition (2008)

11. Gallagher, S.: How the Body Shapes the Mind. Oxford University Press (2005)

12. Scheler, M.: The Nature of Sympathy. New Haven: Yale (original work published 1923), Translated by P. Heath (1954)

13. Bruza, P.D., Cole, R.: Quantum logic of semantic space: An exploratory investigation of context effects in practical reasoning. In Artemov, S., Barringer, H., d'Avila Garcez, A., Woods, J., eds.: We Will Show Them: Essays in Honour of Dov Gabbay, College Publications (2005) 339-361

14. Busemeyer, J.R., Wang, Z., Townsend, J.T.: Quantum dynamics of human decision making. Journal of Mathematical Psychology 50 (2006) 220-242

15. Gabora, L.: The cultural evolution of socially situated cognition. Cognitive Systems Research 9(1-2) (2008) 104-113

16. Gabora, L., Rosch, E., Aerts, D.: Toward an ecological theory of concepts. Ecological Psychology 20(1) (2008) 84-116

17. Kronz, F., Tiehen, J.: Emergence and Quantum Mechanics. Philosophy of Science 69 (2002) 324-347

18. Gallese, V.: The Shared Manifold Hypothesis: From Mirror Neurons to Empathy. Journal of Consciousness Studies 8 (2001) 33-50

19. Hurley, S.: The Shared Circuits Model (SCM): How Control, Mirroring, and Simulation Can Enable Imitation, Deliberation and Mindreading. Behavioral and Brain Sciences 31 (2008) 1-58

20. Jaegher, H.D., Paolo, E.A.D.: Participatory Sense-making: An Enactive Approach to Social Cognition. Phenomenology and the Cognitive Sciences 6(4) (2007) 485 $-507$

21. Gallagher, S., Meltzoff, A.: The Earliest Sense of Self and Others: Merleau-Ponty and Recent Developmental Studies. Philosophical Psychology 9 (1996) 211-233 
22. Thompson, E.: Mind in Life - Biology, Phenomenology and the Sciences of Mind. Harvard University Press (2007)

23. Noë, A.: Action in Perception. MIT Press (2004)

24. Bruza, P., Widdows, D., Woods, J.: A Quantum Logic of Down Below. In: Handbook of Quantum Logic. Amsterdam: Elsevier/North-Holland (2006)

25. Gabbay, D., Woods, J.: The Reach of Abduction: Insight and Trial. Elsevier (2005)

26. Gärdenfors, P.: Conceptual Spaces: The Geometry of Thought. MIT Press (2000)

27. Rizzolatti, G., Fadiga, L., Gallese, V., Fogassi, L.: Premotor cortex and the recognition of motor actions. Cognitive Brain Research 3 (1996) 131-141

28. Fogassi, L., Gallese, V.: The Neural Correlates of Action Understanding in Nonhuman Primates. In: Mirror Neurons and the Evolution of Brain and Language. Amsterdam and Phildadelphia: John Benjamins (2002) 13-35

29. Rizzolatti, G., Craighero, L., Fadiga, L.: The Mirror System in Humans. In: Mirror Neurons and the Evolution of Brain and Language. Amsterdam and Phildadelphia: John Benjamins (2002) 37-62

30. Wohlschläger, A., Bekkering, H.: The Role of Objects in Imitation. In: Mirror Neurons and the Evolution of Brain and Language. Amsterdam and Phildadelphia: John Benjamins (2002) 101-113

31. Gallese, V., Lakoff, G.: The Brain's Concepts: The Role of the Sensory-motor System in Conceptual Knowledge. Cognitive Neuropsychology 22(3/4) (2005) 455-479

32. Husserl, E.: Husserliana 13: Zur Phänomenologie der Intersubjektivität. Texte aus dem Nachlass. Erster Teil: 1905-1920. Den Haag: Martinus Nijhoff (1973)

33. Husserl, E.: Husserliana 15: Zur Phänomenologie der Intersubjektivität. Texte aus dem Nachlass. Dritter Teil: 1929-1935. Den Haag: Martinus Nijhoff (1973)

34. Nadel, J., Carchon, I., Kervella, C., Marcelli, D., Reserbat-Plantey, D.: Expectancies for Social Contingency in Two-month-olds. Developmental Science 2 (1999) $164-173$

35. Auvray, M., Lenay, C., Stewart, J.: Perceptual Interactions in a Minimalist Virtual Environment. New Ideas in Psychology (2008)

36. Husserl, E.: The Crisis of European Sciences and Transcendental Phenomenology: An Introduction to Phenomenological Philosophy. Translated by David Carr. Northwestern University Press (1970)

37. Zahavi, D.: Subjectivity and Selfhood. MIT Press (2006)

38. Heidegger, M.: History of the Concept of Time: Prolegomena. Indiana University Press (1985)

39. Trevarthen, C.: Communication and cooperation in early infancy: A description of primary intersubjectivity. In: Before Speech: The Beginning of Interpersonal Communication. Cambridge University Press (1979) 321-347

40. Trevarthen, C., Hubley, P.: Secondary intersubjectivity: Confidence, confiding and acts of meaning in the first year. In: Action, Gesture and Symbol: The Emergence of Language. London: Academic Press (1978) 183-229

41. Hutto, D., Ratcliffe, M., eds.: Folk Psychology Re-Assessed. Dordrecht: Springer Publishers (2007)

42. Bruner, J.: Actual Minds, Possible Worlds. Harvard University Press (1986) 